\title{
SHEFFER OPERATIONS IN COMPLEMENTED POSETS
}

\author{
IVAN CHAJDA AND MIROSLAV KOLAŘÍK
}

\begin{abstract}
We show that in every downward directed poset with an antitone involution the so-called Sheffer operation can be introduced satisfying certain identities. However, also conversely, if we have given a Sheffer operation $\mid$ on a set $P$, then $P$ can be converted into a poset with an antitone involution ', where both ' and the order relation $\leq$ are derived by $\mid$. Using this, we can characterize orthoposets, i.e. bounded posets with complementation which is an antitone involution by means of identities satisfied by this Sheffer operation. Also conversely, if $\mid$ is a Sheffer operation on a given set $P$ satisfying these identities, then $P$ can be organized into an orthoposet.
\end{abstract}

\section{INTRODUCTION}

The so-called Sheffer operation alias Sheffer stroke was introduced by H. M. Sheffer [5] in 1913 seeking to replace all the operations of a Boolean algebra, i.e. conjunction, disjunction, implication and negation, by only one from which every other can be derived. At present, it is a very useful approach which enables the production of electronic chips with only one sort of so-called diodes, which is technologically easier than the production of various types of these diodes. Hence, the results from 1913 were successfully applied to modern technologies. An introduction to the theory of Boolean operations can be found in the seminal monograph by G. Birkhoff $[1]$.

Because other propositional logics different from the classical one are of interest, namely the logic of quantum mechanics, it raises a question whether such Sheffer operation can be introduced in lattices forming an algebraic semantics of these logics. One attempt was made by the first author [2], when a Sheffer operation was introduced and studied in ortholattices.

However, not every non-classical logic is based on a lattice structure. This calls for research of complemented posets. Such a poset has one unary operation which is a complementation and the order relation. The question is whether, in this case, a Sheffer operation replacing particularly complementation and the order relation can also be defined. It is our aim to define such Sheffer operation on complemented posets and show how its properties can characterize other important properties of the poset in question.

$M S C$ (2010): primary 06A11, 06E30, 03G25.

Keywords: poset, bounded poset, antitone involution, complementation, commutative directoid, Sheffer operation.

The first author is supported by the Austrian Science Fund (FWF), project I 4579-N, and the Czech Science Foundation (GAČR), project 20-09869L, and by IGA, project 2020014. 


\section{Preliminaries}

First, we recall several basic concepts. Let $\mathcal{P}=(P ; \leq)$ be an ordered set (a poset for brief). For $x, y \in P$, we define the so-called upper-cone and lower-cone as follows:

$$
\begin{aligned}
& U(x, y)=\{z \in P ; x \leq z \text { and } y \leq z\}, \\
& L(x, y)=\{w \in P ; w \leq x \text { and } w \leq y\} .
\end{aligned}
$$

A poset $\mathcal{P}$ is downward (upward) directed if $L(x, y) \neq \emptyset$ (or $U(x, y) \neq \emptyset$, respectively) for all $x, y \in P$. If there is an element $0 \in P$ such that $0 \leq x$ for each $x \in P$, then it is called the least element. Dually, if there is an element $1 \in P$ such that $x \leq 1$ for each $x \in P$, then it is called the greatest element. A poset $\mathcal{P}$ is called bounded if it has both 0 and 1 which will be expressed by the notation $\mathcal{P}=(P ; \leq, 0,1)$. Of course, every bounded poset is both downward and upward directed.

A unary operation ' on $P$ is called an involution if $x^{\prime \prime}=x$ for each $x \in P$. It is called antitone if

$$
x \leq y \text { implies } y^{\prime} \leq x^{\prime} .
$$

Let $\mathcal{P}=\left(P ; \leq,{ }^{\prime}, 0,1\right)$ be a bounded poset with a unary operation '. This unary operation is called a complementation if

$$
L\left(x, x^{\prime}\right)=\{0\} \quad \text { and } \quad U\left(x, x^{\prime}\right)=\{1\}
$$

for each $x \in P$; then $\mathcal{P}$ is called a complemented poset. If, moreover, this complementation is an antitone involution, then it is called an orthocomplementation and $\mathcal{P}$ is called an orthoposet. It is evident that if $P$ is a complemented poset, then $x \vee x^{\prime}$ as well as $x \wedge x^{\prime}$ exist for each $x \in P$ and then $x \vee x^{\prime}=1$ and $x \wedge x^{\prime}=0$.

An example of an orthoposet is visualized in Figure 1.

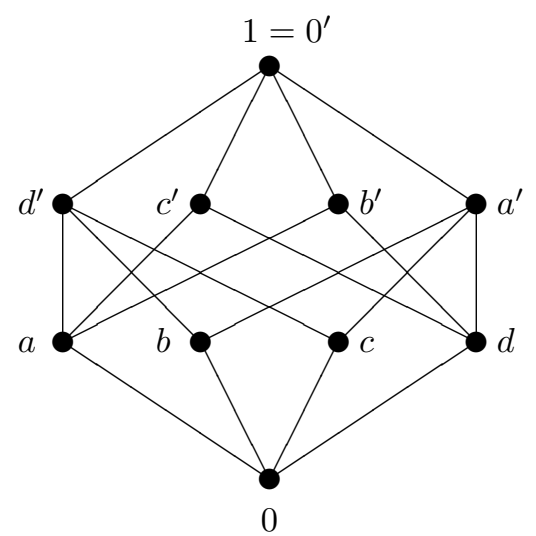

Figure 1. .

Evidently, this poset is not a lattice because, e.g. $\sup (a, d)$ or $\sup (b, c)$ do not exist, we have $U(a, d)=\left\{c^{\prime}, b^{\prime}, 1\right\}, U(b, c)=\left\{a^{\prime}, d^{\prime}, 1\right\}$; dually, $\inf \left(a^{\prime}, d^{\prime}\right)$ and $\inf \left(b^{\prime}, c^{\prime}\right)$ do not exist, we have $L\left(a^{\prime}, d^{\prime}\right)=\{b, c, 0\}$ and $L\left(b^{\prime}, c^{\prime}\right)=\{a, d, 0\}$. 
Let now $\mathcal{P}=(P ; \leq)$ be a downward directed poset. Define an operation $\sqcap$ on $P$ as follows:

- if $x, y$ are comparable, then $x \sqcap y=y \sqcap x=\min (x, y)$,

- if $x, y$ are incomparable, then $x \sqcap y=y \sqcap x$ is an arbitrarily chosen element from $L(x, y)$.

By definition, $x \sqcap x=x, x \sqcap y=y \sqcap x$. It can be easily shown that $\sqcap$ satisfies the so-called weak associativity

$$
x \sqcap((x \sqcap y) \sqcap z)=(x \sqcap y) \sqcap z,
$$

see [3] for details. This groupoid $(P ; \sqcap)$ was introduced by J. Ježek and R. Quackenbush [4] under the name commutative directoid. It is evident that the operation $\sqcap$ need not be determined uniquely, provided $x \wedge y$ does not exist. For example, in the poset depicted in Fig. 1, we can define $b^{\prime} \sqcap c^{\prime}=a$ or $b^{\prime} \sqcap c^{\prime}=d$ or $b^{\prime} \sqcap c^{\prime}=0$. If $\mathcal{P}$ is a given poset, then the commutative directoid reached in this way will be called assigned to $\mathcal{P}$ and will be denoted by $\mathcal{D}(P)=(P ; \sqcap)$.

Also conversely, if $\mathcal{D}=(D ; \sqcap)$ is a commutative directoid, we can define

$$
x \leq y \quad \text { if and only if } \quad x \sqcap y=x .
$$

As pointed out in [3] or [4], $\leq$ is an order on $P$ and then $\mathcal{P}(D)=(D ; \leq)$ will be referred to as an induced poset from $\mathcal{D}$.

What is important for our investigation is that although a commutative directoid $\mathcal{D}(P)=(P ; \sqcap)$ need not be assigned uniquely, when producing the induced poset $\mathcal{P}(\mathcal{D}(P))$, we obtain the original one, i.e.

$$
\mathcal{P}(\mathcal{D}(P))=\mathcal{P} \text {. }
$$

Hence, each of the possible commutative directoids assigned to a poset $\mathcal{P}=(P ; \leq)$ captures all the information from $\mathcal{P}$ and hence it can be converted back to $\mathcal{P}$.

Now, let $\mathcal{P}=\left(P ; \leq,{ }^{\prime}, 0,1\right)$ be a poset with an antitone involution, $\mathcal{D}(P)$ an assigned commutative directoid. It is evident that $0 \sqcap x=0,1 \sqcap x=x$ in $\mathcal{D}(P)$ for every $x \in P$ and that ' is a unary operation in $\mathcal{D}(P)$. This will be expressed by the notation $\mathcal{D}(P)=\left(P ; \sqcap,{ }^{\prime}, 0,1\right)$. Because ' is an antitone involution, we can apply DeMorgan laws to define

$$
x \sqcup y=\left(x^{\prime} \sqcap y^{\prime}\right)^{\prime} .
$$

As shown in $[3],(P ; \sqcup)$ is again a commutative directoid and the absorption laws

$$
x \sqcup(x \sqcap y)=x \quad \text { and } \quad x \sqcap(x \sqcup y)=x
$$

can be easily verified.

\section{SHEFFER OPERATIONS}

Definition 3.1. Let $P$ be a non-empty set. A binary operation $\mid$ on $P$ will be called a Sheffer operation if it satisfies the identities

(i) $x|y=y| x$

(ii) $(x \mid x) \mid(x \mid y)=x$

(iii) $z|((((z \mid y) \mid(z \mid y)) \mid x) \mid(((z \mid y) \mid(z \mid y)) \mid x))=((z \mid y) \mid(z \mid y))| x$. 
In what follows we show that this Sheffer operation is related to posets with an antitone involution.

Theorem 3.2. Let $\mid$ be a Sheffer operation on a given non-empty set P. Define

$$
x^{\prime}=x \mid x
$$

and $x \leq y$ if and only if $x|y=x| x$. Then, $\mathcal{P}=\left(P ; \leq,^{\prime}\right)$ is a poset with an antitone involution.

Proof. From (ii), we obtain $(x \mid x) \mid(x \mid x)=x$; thus, $x^{\prime \prime}=x$, i.e. ' is an involution. The reflexivity of $\leq$ is evident by definition. Assume $x \leq y$ and $y \leq x$. Then, using (i) and (ii), $x|y=x| x, x|y=y| x=y \mid y$; thus,

$$
x=(x \mid x)|(x \mid x)=(y \mid y)|(y \mid y)=y
$$

proves the antisymmetry of $\leq$.

Suppose $x \leq y$ and $y \leq z$. Then, $x|y=x| x, y|z=y| y$ and

$$
\begin{aligned}
x \mid z & =z|x=z|((y \mid x) \mid(y \mid x))=z \mid((((z \mid y) \mid(z \mid y)) \mid x) \mid(((z \mid y) \mid(z \mid y)) \mid x)) \\
& =((z \mid y) \mid(z \mid y))|x=((y \mid y) \mid(y \mid y))| x=y|x=x| x
\end{aligned}
$$

whence $x \leq z$. Altogether, $\leq$ is an order on $P$.

It remains to prove that ' is antitone. Assume $x \leq y$, i.e. $x|y=x| x$. Then, by (i) and (ii), we have

$$
(y \mid y)|(x \mid x)=(y \mid y)|(y \mid x)=y=(y \mid y) \mid(y \mid y)
$$

whence $y^{\prime}=y|y \leq x| x=x^{\prime}$.

Also conversely, in every poset with an antitone involution a Sheffer operation can be defined, see the following result.

Theorem 3.3. Let $\mathcal{P}=\left(P ; \leq,^{\prime}\right)$ be a downward directed poset with an antitone involution. Consider the binary operation $\sqcap$ defined by $(*)$ and define

$$
x \mid y=(x \sqcap y)^{\prime} .
$$

Then, | is a Sheffer operation on $P$.

Proof. (i) is evident by the commutativity of $\sqcap$. By applying the absorption law (Ab), we prove (ii) as follows:

$$
\begin{aligned}
(x \mid x) \mid(x \mid y) & =(x \sqcap x)^{\prime}\left|(x \sqcap y)^{\prime}=x^{\prime}\right|(x \sqcap y)^{\prime}=\left(x^{\prime} \sqcap(x \sqcap y)^{\prime}\right)^{\prime} \\
& =x^{\prime \prime} \sqcup(x \sqcap y)^{\prime \prime}=x \sqcup(x \sqcap y)=x .
\end{aligned}
$$

For (iii) we use the weak associativity of $\sqcap$ :

$$
\begin{aligned}
z \mid((((z \mid y) \mid(z \mid y)) \mid x) \mid(((z \mid y) \mid(z \mid y)) \mid x)) & =z\left|(((z \mid y) \mid(z \mid y)) \mid x)^{\prime}=z\right|((z \sqcap y) \sqcap x) \\
& =(z \sqcap((z \sqcap y) \sqcap x))^{\prime}=((z \sqcap y) \sqcap x)^{\prime} \\
& =(z \sqcap y)\left|x=(z \mid y)^{\prime}\right| x=((z \mid y) \sqcap(z \mid y))^{\prime} \mid x \\
& =((z \mid y) \mid(z \mid y)) \mid x .
\end{aligned}
$$




\section{BOUnded AND COMPLEMENTED POSETS}

In this section, we characterize a bounded poset and an orthoposet by means of the properties of a Sheffer operation.

Lemma 4.1. Let $\mathcal{P}=\left(P ; \leq,{ }^{\prime}, 0,1\right)$ be a bounded poset with an antitone involution and $a$ Sheffer operation | defined by $(* *)$. Then, $(P ; \mid)$ satisfies the following identities

(iv) $x \mid 0=1$

(v) $x|1=x| x$.

Proof. We easily derive that

$$
x \mid 0=(x \sqcap 0)^{\prime}=0^{\prime}=1
$$

and

$$
x\left|1=(x \sqcap 1)^{\prime}=x^{\prime}=x\right| x .
$$

Also conversely, we can prove the following results.

Lemma 4.2. Let $\mid$ be a Sheffer operation on the set $P, 0,1$ be elements of $P$ and (iv) and (v) of Lemma 4.1 hold. Define $x^{\prime}=x \mid x$ and $x \leq y$ if and only if $x|y=x| x$. Then, $\mathcal{P}=\left(P ; \leq,{ }^{\prime}, 0,1\right)$ is a bounded poset with an antitone involution.

Proof. Using Theorem 3.2, we only need to verify that $x \sqcup 0=x, x \sqcup 1=1$, $x \sqcap 0=0$ and $x \sqcap 1=x$. First, we can see that $0^{\prime}=1$ and $1^{\prime}=0$ because $0 \mid 0=1$ hold by (iv) of Lemma 4.1 and $1 \mid 1=0$ if and only if $(0 \mid 0) \mid(0 \mid 0)=0$ hold by (ii) of Definition 3.1. Further, for all $x \in P$, we have $0 \leq x$ by (iv) of Lemma 4.1 and $x \leq 1$ by $(\mathrm{v})$ of Lemma 4.1. Since $0 \leq x \leq 1$, we have immediately $x \sqcup 0=x$, $x \sqcup 1=1, x \sqcap 0=0$ and $x \sqcap 1=x$.

We are going to show that the property of complementation in a bounded poset can also be expressed by identities satisfied by a Sheffer operation.

Theorem 4.3. Let 0,1 be elements of a given set $P$ and $\mid$ be a Sheffer operation on $P$ satisfying identities (iv) and (v). Moreover, let $\mid$ satisfy identities

(vi) $(((x \mid x) \mid(y \mid y)) \mid((x \mid x) \mid(y \mid y))) \mid((x \mid(y \mid y)) \mid(x \mid(y \mid y)))=1$

(vii) $(((x \mid y) \mid(x \mid y)) \mid(((x \mid x) \mid y) \mid((x \mid x) \mid y))) \mid(((x \mid y) \mid(x \mid y)) \mid(((x \mid x) \mid y) \mid((x \mid x) \mid y)))=0$.

Define $x^{\prime}=x \mid x$ and $x \leq y$ if and only if $x|y=x| x$. Then, $\mathcal{P}=\left(P ; \leq,{ }^{\prime}, 0,1\right)$ is an orthoposet.

Proof. We easily derive that $x \sqcap y:=(x \mid y) \mid(x \mid y)$ and $x \sqcup y:=(x \mid x) \mid(y \mid y)$. Using Theorem 3.2 and Lemma 4.2, we only need to prove that $\mathcal{P}$ is complemented. Suppose that $\mathcal{P}$ satisfies identities (vi) and (vii). If $x, x^{\prime} \leq y$, then $x \sqcup y=x^{\prime} \sqcup y=y$ and hence

$$
\begin{aligned}
y & =y \sqcup y=(x \sqcup y) \sqcup\left(x^{\prime} \sqcup y\right)=((x \mid x) \mid(y \mid y)) \sqcup(x \mid(y \mid y)) \\
& =(((x \mid x) \mid(y \mid y)) \mid((x \mid x) \mid(y \mid y))) \mid((x \mid(y \mid y)) \mid(x \mid(y \mid y)))=1
\end{aligned}
$$

showing $x \vee x^{\prime}=1$. 
Similarly, $y \leq x, x^{\prime}$ implies $x \sqcap y=x^{\prime} \sqcap y=y$ and therefore

$$
\begin{aligned}
y & =y \sqcap y=(x \sqcap y) \sqcap\left(x^{\prime} \sqcap y\right)=((x \mid y) \mid(x \mid y)) \sqcap(((x \mid x) \mid y) \mid((x \mid x) \mid y)) \\
& =(((x \mid y) \mid(x \mid y)) \mid(((x \mid x) \mid y) \mid((x \mid x) \mid y))) \mid(((x \mid y) \mid(x \mid y)) \mid(((x \mid x) \mid y) \mid((x \mid x) \mid y)))=0
\end{aligned}
$$

showing $x \wedge x^{\prime}=0$. Hence, $\mathcal{P}$ is complemented.

The converse of Theorem 4.3 is also true, see the following.

Theorem 4.4. Let $\mathcal{P}=\left(P, \leq,{ }^{\prime}, 0,1\right)$ be an orthoposet and $\mid$ be the operation on $P$ defined by (**). Then, $\mid$ is a Sheffer operation on $P$ satisfying identities (iv)-(vii).

Proof. Using Lemma 4.1, we only need to verify (vi) and (vii). Let $x, y \in P$ and assume $\mathcal{P}$ is bounded and complemented. Then,

$$
\begin{gathered}
x \leq x \sqcup y \leq(x \sqcup y) \sqcup\left(x^{\prime} \sqcup y\right), \\
x^{\prime} \leq x^{\prime} \sqcup y \leq(x \sqcup y) \sqcup\left(x^{\prime} \sqcup y\right)
\end{gathered}
$$

and hence $(x \sqcup y) \sqcup\left(x^{\prime} \sqcup y\right) \in U\left(x, x^{\prime}\right)=\{1\}$. So,

$$
\begin{aligned}
1 & =(x \sqcup y) \sqcup\left(x^{\prime} \sqcup y\right) \\
& =(x \sqcup y)^{\prime}\left|\left(x^{\prime} \sqcup y\right)^{\prime}=\left(x^{\prime} \mid y^{\prime}\right)^{\prime}\right|\left(x \mid y^{\prime}\right)^{\prime} \\
& =\left(\left(x^{\prime} \mid y^{\prime}\right) \mid\left(x^{\prime} \mid y^{\prime}\right)\right) \mid\left(\left(x \mid y^{\prime}\right) \mid\left(x \mid y^{\prime}\right)\right) \\
& =(((x \mid x) \mid(y \mid y)) \mid((x \mid x) \mid(y \mid y))) \mid((x \mid(y \mid y)) \mid(x \mid(y \mid y))),
\end{aligned}
$$

which proves (vi).

Further,

$$
\begin{aligned}
& (x \sqcap y) \sqcap\left(x^{\prime} \sqcap y\right) \leq x \sqcap y \leq x, \\
& (x \sqcap y) \sqcap\left(x^{\prime} \sqcap y\right) \leq x^{\prime} \sqcap y \leq x^{\prime}
\end{aligned}
$$

and hence $(x \sqcap y) \sqcap\left(x^{\prime} \sqcap y\right) \in L\left(x, x^{\prime}\right)=\{0\}$. Thus,

$$
\begin{aligned}
0 & =(x \sqcap y) \sqcap\left(x^{\prime} \sqcap y\right) \\
& =\left((x \sqcap y) \mid\left(x^{\prime} \sqcap y\right)\right)^{\prime} \\
& =\left((x \mid y)^{\prime} \mid\left(x^{\prime} \mid y\right)^{\prime}\right) \mid\left((x \mid y)^{\prime} \mid\left(x^{\prime} \mid y\right)^{\prime}\right) \\
& =(((x \mid y) \mid(x \mid y)) \mid(((x \mid x) \mid y) \mid((x \mid x) \mid y))) \mid(((x \mid y) \mid(x \mid y)) \mid(((x \mid x) \mid y) \mid((x \mid x) \mid y)))
\end{aligned}
$$

which proves (vii).

\section{REFERENCES}

[1] G. Birkhoff, Lattice Theory, 3rd ed., Proc. Amer. Math. Soc., Providence, R.I., 1967.

[2] I. Chajda, Sheffer operation in ortholattices, Acta Univ. Palack. Olomuc. Fac. Rerum Natur Math. 44 (2005), 19-23.

[3] I. Chajda and H. Länger, Directoids. An Algebraic Approach to Ordered Sets, Heldermann, Lemgo, 2011.

[4] J. Ježek and R. Quackenbush, Directoids: Algebraic models of up-directed sets, Algebra Universalis 27 (1990), 49-69.

[5] H. M. Sheffer, A set of five independent postulates for Boolean algebras, with application to logical constants, Trans. Amer. Math. Soc. 14 (1913), 481-488. 
Ivan Chajda, Department of Algebra and Geometry, Palacký University Olomouc, Faculty of Science, 17. listopadu 12, 77146 Olomouc, Czech Republic e-mail: ivan.chajda@upol.cz

Miroslav Kolařík, Department of Computer Science, Palacký University Olomouc, Faculty of Science, 17. listopadu 12, 77146 Olomouc, Czech Republic

e-mail: miroslav.kolarik@upol.cz 
